\title{
Cartan Subalgebras, Weight Spaces, and Criterion of Solvability of Finite Dimensional Leibniz Algebras
}

\author{
Sergio A. Albeverio, Shavkat A. Ayupov, \\ and Bakhrom A. OMIROV
}

\author{
Institut für Angewandte Mathematik \\ Universität Bonn \\ Wegelstr. 6 \\ D-53115 Bonn - Germany \\ albeverio@uni-bonn.de
}

\author{
Institute of Mathematics \\ Uzbekistan Academy of Science \\ F. Hodjaev str. 29 \\ 700143, Tashkent — Uzbekistan \\ e_ayupov@hotmail.com omirovb@mail.ru
}

Received: June 23, 2005

Accepted: November 7, 2005

\section{ABSTRACT}

In this work the properties of Cartan subalgebras and weight spaces of finite dimensional Lie algebras are extended to the case of Leibniz algebras. Namely, the relation between Cartan subalgebras and regular elements are described, also an analogue of Cartan's criterion of solvability is proved.

Key words: Leibniz algebra, Cartan subalgebra, weight space.

2000 Mathematics Subject Classification: 17A32, 17A60, 17B30.

\section{Introduction}

The present work is devoted to the investigation of the Leibniz algebras, which were introduced by J.-L. Loday in [10] and considered further in works [5-8,11].

In studying the properties of the homology of Lie algebras Loday noted that if in the definition of an $n$-th chain the exterior product is changed by the tensor product then in order to prove the derived property defined on chains it is sufficient

This work is supported in part by the DFG 436 USB 113/4 project (Germany) and the Fundamental Science Foundation of Uzbekistan. 
that the algebra satisfies the Leibniz identity instead of antisymmetricity and Jacobi identities. This motivated the introduction of Leibniz algebras, which are a "non skewsymmetric" generalization of Lie algebras. For Leibniz algebras a natural problem arises - to prove analogues of theorems from the theory of Lie algebras.

In the structure theory of finite dimensional Lie algebras it is known that an arbitrary Lie algebra is decomposed into the direct sum of solvable and semisimple parts. In Malcev's work [12] it was shown that the description of solvable Lie algebras is reduced to the description of nilpotent algebras.

Investigations of nilpotent Leibniz algebras [1-3] show that many nilpotent properties of Lie algebras can be extended to the case of nilpotent Leibniz algebras.

In the structure theory of Lie algebras the crucial role is played by Cartan subalgebras and the decomposition of algebras into weight (root) spaces with respect to Cartan (or nilpotent) subalgebras.

In non-Lie Leibniz algebras the ideal generated by squares of elements of the algebra is very important. It is easy to see that this ideal for such algebras is abelian and non trivial.

In the present paper we consider a similar approach to the investigation of Cartan subalgebras and weight spaces of Leibniz algebras.

\section{Preliminaries}

Definition 1.1. An algebra $L$ over a field $F$ is called Leibniz algebra if for any $x, y, z \in L$ the Leibniz identity

$$
[x,[y, z]]=[[x, y], z]-[[x, z], y]
$$

holds, where $[\cdot, \cdot]$ is a bilinear multiplication in $L$.

Note that if in $L$ the identity $[x, x]=0$ holds, then the Leibniz and Jacobi identities coincide. Thus, Leibniz algebras are a "noncommutative" analogue of Lie algebras.

For an arbitrary algebra we define the sequences:

(i) $L^{[1]}=L, L^{[n+1]}=\left[L^{[n]}, L^{[n]}\right]$;

(ii) $L^{1}=L, L^{n+1}=\left[L^{1}, L^{n}\right]+\left[L^{2}, L^{n-1}\right]+\cdots+\left[L^{n-1}, L^{2}\right]+\left[L^{n}, L^{1}\right]$.

Definition 1.2. An algebra $L$ is called solvable if there exists $m \in N$ such that $L^{[m]}=0$.

An algebra $L$ is called nilpotent if there exists $s \in N$ such that $L^{s}=0$.

For an arbitrary element $x$ of $L$ we consider the operator of right multiplication $R_{x}: L \rightarrow L$, where $R_{x}(z)=[z, x]$. The set $R(L)=\left\{R_{x}: x \in L\right\}$ forms a Lie algebra with respect to the operation of commutating, and the following identity holds:

$$
R_{x} R_{y}-R_{y} R_{x}=R_{[y, x]}
$$


From this identity it is easy to see that the solvability of the Lie algebra $R(L)$ is equivalent to the solvability of the Leibniz algebra $L$.

Further in this paper we will assume that all algebras, vector spaces and modules are finite dimensional.

The following lemma gives the decomposition of a vector space into the direct sum of invariant subspaces with respect to a linear transformation.

Lemma 1.3 (Fitting's lemma). Let $V$ be a vector space and $A: V \rightarrow V$ be a linear transformation. Then $V=V_{0 A} \oplus V_{1 A}$, where $A\left(V_{0 A}\right) \subseteq V_{0 A}, A\left(V_{1 A}\right) \subseteq V_{1 A}$ and $V_{0 A}=\left\{v \in V \mid A^{i}(v)=0\right.$ for some $\left.i\right\}, V_{1 A}=\bigcap_{i=1}^{\infty} A^{i}(V)$. Moreover, $A_{\mid V_{0 A}}$ is a nilpotent transformation and $A_{\mid V_{1 A}}$ is an automorphism.

Proof. See [9, chapter II, §4].

Definition 1.4. The spaces $V_{0 A}$ and $V_{1 A}$ are called respectively Fitting null component and Fitting one component of the space $V$ with respect to the transformation $A$.

Lemma 1.5. Let $V$ be a vector space. And let $A, B$ be linear transformations of $V$ such that

$$
[\cdots[[B, \underbrace{A], A], \ldots, A}_{k \text { times }}]=0 .
$$

Then the Fitting components $V_{0 A}, V_{1 A}$ of the space $V$ with respect to $A$ are also invariant with respect to the transformation $B$.

Proof. See [9, chapter II, §4].

Let $L$ be a nilpotent Leibniz algebra, then it is evident that the Lie algebra $R(L)$ is also nilpotent. Further we will use the following results:

Theorem 1.6. Let $L$ be a nilpotent Lie algebra of linear transformations of a vector space $V$ and $V_{0}=\bigcap_{A \in L} V_{0 A}, V_{1}=\bigcap_{i=1}^{\infty} L^{i}(V)$. Then the subspaces $V_{0}$ and $V_{1}$ are invariant with respect to $L$ (i.e., $V_{0}$ and $V_{1}$ are invariant with respect to every transformation $B$ from $L$ ) and $V=V_{0} \oplus V_{1}$. Moreover, $V_{1}=\sum_{A \in L} V_{1 A}$.

Proof. See [9, chapter II, §4].

Theorem 1.7. Let $G$ be a split nilpotent Lie algebra of linear transformations of a vector space $M$. Then $G$ has a finite number of different weights, weight subspaces are submodules of $M$, and $M$ is decomposed into the direct sum of these modules. Moreover, if $M=M_{1} \oplus M_{2} \oplus \cdots \oplus M_{r}$ is an arbitrary decomposition of $M$ into the sum of subspaces $M_{i}(\neq 0)$, which are invariant with respect to $G$ such that the following conditions hold:

(i) for each $i$ the restriction of $A \in G$ on $M_{i}$ has only one characteristic root $\alpha_{i}(A)$ (of some multiplicity); 
(ii) if $i \neq j$, then there exists $A \in G$ such that $\alpha_{i}(A) \neq \alpha_{j}(A)$;

then the maps $A \rightarrow \alpha_{i}(A)$ are weights and $M_{i}$ are weight subspaces.

Proof. See [9, theorem 7, page 43].

Proposition 1.8. Let $\aleph$ be a Lie algebra of linear transformations of a vector space over a field of zero characteristic, $G$ be the radical (solvable) of the algebra $\aleph$ and $\mathfrak{R}$ be the radical of the associative algebra $\aleph^{*}$. Then $\aleph \cap \Re$ consists of all nilpotent elements of the radical $G$ and $[G, \aleph] \subseteq \mathfrak{R}$. ( $\mathfrak{R}$ is considered as a nilpotent radical of the associative algebra $\aleph^{*}$.)

Proof. See [9, chapter II].

\section{Cartan subalgebras of finite dimensional Leibniz algebras}

Let $\mathfrak{I}$ be a nilpotent subalgebra of a Leibniz algebra $L$ and $L=L_{0} \oplus L_{1}$ be the Fitting decomposition of the algebra $L$ with respect to the nilpotent Lie algebra $R(\mathfrak{I})=\left\{R_{x} \mid x \in \mathfrak{I}\right\}$ of transformations of the vector space $L$ according to theorem 1.6.

The set $l(\mathfrak{I})=\{x \in L \mid[x, \mathfrak{I}] \subseteq \mathfrak{I}\}$ is called left normalizator of the subalgebra $\mathfrak{I}$ in the algebra $L$.

The set $r(\mathfrak{I})=\{x \in L \mid[\mathfrak{I}, x] \subseteq \mathfrak{I}\}$ is called right normalizator of the subalgebra $\mathfrak{I}$ in the algebra $L$.

Definition 2.1. A subalgebra $\mathfrak{I}$ of a Leibniz algebra $L$ is called Cartan subalgebra if the following two conditions are satisfied:

(i) $\mathfrak{I}$ is nilpotent;

(ii) $\mathfrak{I}$ coincides with the left normalizator of $\mathfrak{I}$ in the algebra $L$.

Since in the Lie algebras case we have antisymmetricity, the sets $l(\mathfrak{I})$ and $r(\mathfrak{I})$ obviously coincide. For a Cartan subalgebra of the Leibniz algebra we have only $l(\mathfrak{I}) \subseteq r(\mathfrak{I})$. It is easy to see that if $\mathfrak{I}$ contains the ideal generated by squares of elements of the algebra $L$, then we have $l(\mathfrak{I})=r(\mathfrak{I})$. In non-Lie Leibniz algebras the non coincidence of these sets in general follows from the following example.

Example 2.2. Let $L$ be the Leibniz algebra defined by the following multiplication:

$$
[x, z]=x, \quad[z, y]=y, \quad[y, z]=-y, \quad[z, z]=x,
$$

where $\{x, y, z\}$ is the basis of $L$ and omitted products are equal to zero. Then $\mathfrak{I}=\{x-z\}$ is the Cartan subalgebra of the algebra $L$, but $r(\mathfrak{I})=\{x, z\}$.

Proposition 2.3. A nilpotent subalgebra $\mathfrak{I}$ of a Leibniz algebra $L$ is a Cartan subalgebra if and only if $\mathfrak{I}$ coincides with $L_{0}$ in the Fitting decomposition of the algebra $L$ with respect to $R(\mathfrak{I})$. 
Proof. Firstly, we note that $l(\mathfrak{I}) \subseteq L_{0}$. In fact, if $x \in l(\mathfrak{I})$ then $[x, h] \in \mathfrak{I}$ for any $h \in \mathfrak{I}$. Since the subalgebra $\mathfrak{I}$ is nilpotent, there exists $k \in N$ such that

$$
\left[\cdots \left[[x, \underbrace{h], h], \ldots, h}_{k \text { times }}]=R_{h}^{k}(x)=0,\right.\right.
$$

this implies that $x \in L_{0}$, i.e., $l(\mathfrak{I}) \subseteq L_{0}$. Since $\mathfrak{I} \subseteq l(\mathfrak{I})$ we have $\mathfrak{I} \subseteq L_{0}$.

Suppose that $\mathfrak{I} \subset L_{0}\left(\mathfrak{I} \neq L_{0}\right)$. By theorem 1.6, the space $L_{0}$ is invariant with respect to $R(\mathfrak{I})$ and the restriction of the operator $R_{h}$ on $L_{0}$, where $h \in \mathfrak{I}$, is nilpotent. Moreover, $\mathfrak{I}$ is an invariant subspace of the space $L_{0}$ with respect to $R(\mathfrak{I})$. Thus, we obtain the induced Lie algebra $\overline{\mathfrak{I}}$ of linear transformations which acts on the non null space $L_{0} / \mathfrak{I}$. Since these transformations are nilpotent then by a version of Engel's theorem we have that $\overline{\mathfrak{I}}(x+\mathfrak{I})=\overline{0}$, where $x+\mathfrak{I}$ a non zero vector. It means that the condition $[x, h] \in \mathfrak{I}$ is verified for any $h \in \mathfrak{I}$; therefore $x \in l(\mathfrak{I})$ and $x \notin \mathfrak{I}$, so $\mathfrak{I} \neq l(\mathfrak{I})$. Thus, $\mathfrak{I} \subset l(\mathfrak{I})$ if and only if $\Im \subset L_{0}$, and the assertion is proved.

Proposition 2.4. Let $\mathfrak{I}$ be a nilpotent subalgebra of a Leibniz algebra L, and let $L=L_{0} \oplus L_{1}$ be the Fitting decomposition of the algebra $L$ with respect to $R(\mathfrak{I})$. Then $L_{0}$ is a subalgebra and $\left[L_{1}, L_{0}\right] \subseteq L_{1}$.

Proof. Let $h \in \mathfrak{I}, a \in L_{0}$. Then there exists $k \in N$ such that

$$
[\cdots[a, \underbrace{h], h], \ldots, h]}_{k \text { times }}=0 .
$$

From this we have $\left[\cdots\left[\left[R_{a}, R_{h}\right], R_{h}\right], \ldots, R_{h}\right]=(-1)^{k} R_{[\cdots[a, h], h], \ldots, h]}=0$. From this relation and lemma 1.5 it follow that the Fitting subspaces $L_{0 R_{h}}$ and $L_{1 R_{h}}$ of the algebra $\mathfrak{I}$ which correspond to the endomorphism $R_{h}$ are invariant subspaces with respect to $R_{a}$. Since $L_{0}=\bigcap_{h \in \mathfrak{I}} L_{0 R_{h}}$ and $L_{1}=\bigcap_{h \in \mathfrak{I}} L_{1 R_{h}}$, then $R_{a}\left(L_{0}\right) \subseteq L_{0}$ and $R_{a}\left(L_{1}\right) \subseteq L_{1}$. And since $a$ is an arbitrary element in $L_{0}$, we obtain $\left[L_{0}, L_{0}\right] \subseteq L_{0}$ and $\left[L_{1}, L_{0}\right] \subseteq L_{1}$.

Definition 2.5. An element $h$ of $L$ is called regular if the dimension of the Fitting null component of the Leibniz algebra $L$ with respect to $R_{h}$ is minimal. This dimension is called the rank of the algebra $L$.

The set $Z(L)=\{x \in L \mid[L, x]=0\}$ will be called right annihilator of the algebra $L$. It is easy to see that the dimension of the Fitting null component of a linear transformation $A$ is equal to the order of the zero root of the characteristic polynomial of its transformation.

Therefore an element $h$ is regular if and only if the order of the zero characteristic root for $R_{h}$ is minimal.

Note that in the Lie algebras case the linear transformation $R_{h}$ is degenerated since $[h, h]=0$ for any $h$, and therefore the rank of the Lie algebra is greater than zero. 
The following lemma shows that in the Leibniz algebras case the rank is also greater than zero.

Lemma 2.6. Let $L$ be a Leibniz algebra. Then operator $R_{x}$ is degenerated for any $x$ in $L$.

Proof. Suppose that the algebra $L$ is a non Lie algebra. And suppose that there exists a non zero $x$ in $L$ such that $R_{x}$ is not degenerated. Then $[L, x]=L$ and therefore, there exists a non null element $y$ of algebra $L$ such that $[y, x]=x$. Since $I^{\text {ann }}=\operatorname{ideal}\langle[a, a] \mid a \in L\rangle \subseteq Z(L)$, then it is easy to see that $\{x, y,[x, y]\} \not \subset I^{\text {ann }}$.

Let the dimension of the algebra $L$ be equal to $n$. Put $x_{1}:=y, x_{2}:=x, x_{3}:=$ $\left[x_{2}, x_{2}\right], \ldots, x_{n+1}:=\left[x_{n}, x_{2}\right]$. Since the operator $R_{x}$ is not degenerated we have $x_{i} \neq 0$ for any $i=3, \ldots, n+1$ and every $x_{i}$ is contained in $I^{\text {ann }}$. Let us show that the system $\left\{x_{1}, x_{2}, \ldots, x_{n+1}\right\}$ is linearly independent.

The elements $x_{1}$ and $x_{2}$ are linearly independent. In fact, otherwise $y=\beta x$, where $\beta$ is different from zero. Then $x=[y, x]=\beta[x, x]=\beta[x,[y, x]]=\beta^{2}[x,[x, x]]=0$, and we obtain contradiction with a condition $x \neq 0$.

Suppose that $\alpha_{1} x_{1}+\alpha_{2} x_{2}+\cdots+\alpha_{n+1} x_{n+1}=0$ for appropriate $\alpha_{i}, i=1, \ldots, n+1$. Multiplying this equation from the left hand side by the element $x_{2}$, we obtain $\alpha_{1}\left[x_{2}, x_{1}\right]+\alpha_{2} x_{3}=0$. Since $\left[x_{2}, x_{1}\right]$ does not belong to the ideal $I^{\text {ann }}$, unlike $x_{3}$, then $\alpha_{1}=\alpha_{2}=0$. Consider the equation $\alpha_{3} x_{3}+\alpha_{4} x_{4}+\cdots+\alpha_{n+1} x_{n+1}=0$, which we can rewrite in the following form: $\alpha_{3}\left[x_{2}, x_{2}\right]+\alpha_{4}\left[x_{3}, x_{2}\right]+\cdots+\alpha_{n+1}\left[x_{n}, x_{2}\right]=$ $R_{x}\left(\alpha_{3} x_{2}+\alpha_{4} x_{3}+\cdots+\alpha_{n+1} x_{n}\right)=0$. And again using the fact that $R_{x}$ is non degenerated we have that $\alpha_{3} x_{2}+\alpha_{4} x_{3}+\cdots+\alpha_{n+1} x_{n}=0$. Since $x_{2}$ does not belong to the ideal $I^{\text {ann }}$, unlike $\alpha_{4} x_{3}+\cdots+\alpha_{n+1} x_{n}$, we have $\alpha_{3}=0$. Continuing similarly we obtain that $\alpha_{4}=\alpha_{5}=\cdots=\alpha_{n+1}=0$, i.e., the system $\left\{x_{1}, x_{2}, \ldots, x_{n+1}\right\}$ is linearly independent. This is in contradiction with the dimension of the algebra $L$.

The following theorem establishes relations between regular elements of Leibniz algebra and Cartan subalgebras.

Theorem 2.7. Let $L$ be a Leibniz algebra over a infinite field $F$ and a be a regular element of $L$. Then the Fitting null component $\mathfrak{I}$ of algebra $L$ with respect to $R_{a}$ is a Cartan subalgebra.

Proof. Let $L=\mathfrak{I} \oplus \mathfrak{R}$ be a Fitting decomposition of $L$ with respect to $R_{a}$. As it will shown below one may assume without loss of generality that the one-generated subalgebra $\langle a\rangle$ is nilpotent. Then by proposition 2.4 we have that $\mathfrak{I}$ is a subalgebra and $[\Re, \mathfrak{I}] \subseteq \mathfrak{I}$. Now we prove that any transformation $R_{b \mid \mathfrak{I}}$, where $b \in \mathfrak{I}$, is nilpotent. Otherwise, let $b \in \mathfrak{I}$ be an element such that $R_{b \mid \mathfrak{I}}$ is not nilpotent. Choose a basis in $L$ consisting of bases of $\mathfrak{I}$ and $\mathfrak{R}$. The matrix of $R_{h}, h \in \mathfrak{I}$, in this basis has the form $\left(\begin{array}{cc}\left(\rho_{1}\right) & 0 \\ 0 & \left(\rho_{2}\right)\end{array}\right)$, where $\left(\rho_{1}\right)$ is the matrix of $R_{h \mid \mathfrak{I}}$ and $\left(\rho_{2}\right)$ is the matrix of $R_{h \mid \Re}$.

Let $A=\left(\begin{array}{cc}\left(\alpha_{1}\right) & 0 \\ 0 & \left(\alpha_{2}\right)\end{array}\right)$ and $B=\left(\begin{array}{cc}\left(\beta_{1}\right) & 0 \\ 0 & \left(\beta_{2}\right)\end{array}\right)$ be the matrices of $R_{a}$ and $R_{b}$, respectively. Since $\left(\alpha_{2}\right)$ is not degenerated we have $\operatorname{det}\left(\alpha_{2}\right) \neq 0$. By hypothesis, 
the matrix $\left(\beta_{1}\right)$ is not nilpotent. Therefore if $l=\operatorname{rank} L$ then $\operatorname{dim} \mathfrak{I}=l$ and the characteristic polynomial of the matrix $\left(\beta_{1}\right)$ is not divisible by $\lambda^{l}$. Let $\lambda, \mu$, $\nu$ be algebraic independent variables and let $P(\lambda, \mu, \nu)$ be the characteristic polynomial, i.e., $P(\lambda, \mu, \nu)=\operatorname{det}(\lambda 1-\mu A-\nu B)=\operatorname{det}(\lambda 1-(\mu A+\nu B))$. Then the equality $P(\lambda, \mu, \nu)=P_{1}(\lambda, \mu, \nu) P_{2}(\lambda, \mu, \nu)$, where $P_{i}(\lambda, \mu, \nu)=\operatorname{det}\left(\lambda_{1}-\mu\left(\alpha_{i}\right)-\nu\left(\beta_{i}\right)\right)=$ $\operatorname{det}\left(\lambda 1-\left(\mu\left(\alpha_{i}\right)+\nu\left(\beta_{i}\right)\right)\right)$ holds. As it was noted above the polynomial $P_{2}(\lambda, 1,0)=$ $\operatorname{det}\left(\lambda 1-\left(\alpha_{2}\right)\right)$ is not divisible by $\lambda$ and the polynomial $P_{1}(\lambda, 0,1)=\operatorname{det}\left(\lambda 1-\left(\beta_{1}\right)\right)$ is not divisible by $\lambda^{l}$. Therefore the greatest degree of $\lambda$ on which the polynomial $P(\lambda, \mu, \nu)$ can be divided is $\lambda^{l^{\prime}}$, where $l^{\prime}<l$. Since the field $F$ is infinite, we can choose $\mu_{0}$ and $\nu_{0}$ such that $P\left(\lambda, \mu_{0}, \nu_{0}\right)$ is not divisible by $\lambda^{l^{\prime}+1}$. Put $c:=\mu_{0} a+\nu_{0} b$, then the characteristic polynomial $\operatorname{det}\left(\lambda 1-R_{c}\right)=\operatorname{det}\left(\lambda 1-\mu_{0} A-\nu_{0} B\right)=P\left(\lambda, \mu_{0}, \nu_{0}\right)$ is not divisible by $\lambda^{l^{\prime}+1}$. Therefore the order of zero root for $R_{c}$ will be equal to $l^{\prime}<l$. But $a$ is a regular element. Thus, for any $b \in \mathfrak{I}$ the operator $R_{b \mid \mathfrak{I}}$ is nilpotent. Using now Engel's theorem [4] we obtain that $\mathfrak{I}$ is a nilpotent Leibniz algebra. Let $L_{0}$ be a Fitting null-component of the algebra $L$ with respect to $R(\mathfrak{I})$. Since $\mathfrak{I}$ is the Fitting null component of the transformation $R_{a}$, then $L_{0} \subseteq \mathfrak{I}$. Indeed, when $a \in \mathfrak{I}$ we have that $L_{0}=\bigcap_{b \in \mathfrak{I}} L_{0 R_{b}} \subseteq L_{0 R_{a}}$.

Let $a \notin \mathfrak{I}$. Then $a^{k} \neq 0$ for any $k \in N$. Consider the following elements:

$$
a_{1}:=a, \quad a_{2}:=[a, a], \quad \ldots, \quad a_{n+1}:=[\underbrace{[[a, a], a], \ldots, a]}_{n+1 \text { times }}=0
$$

where $n=\operatorname{dim} L$. These elements are not equal to zero but linearly dependent, i.e., there exists a non trivial linear combination

$$
\alpha_{1} a_{1}+\alpha_{2} a_{2}+\cdots+\alpha_{n+1} a_{n+1}=0 .
$$

Let $\alpha_{1} \neq 0$, then using the fact that $a_{i} \in I^{\text {ann }}=\operatorname{ideal}\langle[x, x] \mid x \in L\rangle$ for any $2 \leq i \leq n+1$, we obtain that $a_{1} \in I^{\text {ann }} \subseteq Z(L)$ and consequently $a_{2}=0$, i.e., we have a contradiction with the condition $a_{2} \neq 0$. Thus, $\alpha_{1}=0$.

Let $k$ be the minimal number, such that $\alpha_{k} \neq 0$. Then $\alpha_{k} a_{k}+\cdots+\alpha_{n+1} a_{n+1}=0$ and therefore for the element $t=\alpha_{k} a_{1}+\cdots+\alpha_{n+1} a_{n+1-k}$ we have that

$$
[\underbrace{[[t, t], t], \ldots, t]}_{k \text { times }}=0
$$

and $R_{t}=\alpha_{k} R_{a}$, i.e., $t$ is a regular element and $L_{0 R_{a}}=L_{0 R_{t}}=\mathfrak{I}$. On the other hand, $L_{0} \supseteq \mathfrak{I}$ for any nilpotent subalgebra $\mathfrak{I}$. In fact, $L_{0}=\bigcap_{b \in \mathfrak{I}} L_{0 R_{b}}$ and if $h \in \mathfrak{I}$ then

$$
\underbrace{[[[h, b], b], \ldots, b]}_{s \text { times }}=0
$$

for any $b \in \mathfrak{I}$ (here $s$ is the index of nilpotence of the algebra $\mathfrak{I}$ ) thus $h \in L_{0}$ and $L_{0}=\mathfrak{I}$. Using proposition 2.3 we obtain that $\mathfrak{I}$ is a Cartan subalgebra, which completes the proof of the theorem. 
Another useful remark about regular elements and Cartan subalgebras is the following: if a Cartan subalgebra contains a regular element $a$, then $\mathfrak{I}$ is uniquely defined by the element $a$ as Fitting null component of the algebra $L$ with respect to $R_{a}$, i.e., $\mathfrak{I}=L_{0 R_{a}}$.

In fact, if we denote $L_{0 R_{a}}$ by $\mathfrak{R}$ then it is evident that $\mathfrak{I} \subseteq \mathfrak{R}$, since $\mathfrak{I}$ is nilpotent. (If $h \in \mathfrak{I}$ then using the nilpotence of $\mathfrak{I}$ and from $a \in \mathfrak{I}$ we have $[[[h, a], a], \ldots, a]=0$ and then $h \in L_{0 R_{a}}=\Re$.)

On the other hand, from theorem 1.6 we have that $\mathfrak{R}$ is nilpotent. And if $\mathfrak{I} \neq \mathfrak{R}$ then there exists $z \in \mathfrak{R} \backslash \mathfrak{I}$. If $[z, \mathfrak{I}] \subseteq \mathfrak{I}$, since $\mathfrak{I}$ is a Cartan subalgebra, we have $z \in \mathfrak{I}$, a contradiction.

Therefore for any $z \in \mathfrak{R} \backslash \mathfrak{I}$ we have $[z, \mathfrak{I}] \not \subset \mathfrak{I}$. Then there exist $l_{1}, l_{2}, \ldots, l_{k} \in \mathfrak{I}$ such that $\left[\left[\left[z, l_{1}\right], l_{2}\right], \ldots, l_{k}\right] \in \mathfrak{R} \backslash \mathfrak{I}$ and $\left[\left[\left[z, l_{1}\right], l_{2}\right], \ldots, l_{k}\right] \neq 0$, i.e., $\mathfrak{R}$ is not nilpotent, also a contradiction. Therefore $\mathfrak{I}=\mathfrak{R}$.

From this it follows that two Cartan subalgebras having the same regular element coincide.

For the Leibniz algebra $L$, we consider the natural homomorphism $\varphi$ into the factor algebra $L_{\text {Lie }}$, where $L_{\text {Lie }}=L / I^{\text {ann }}$

Proposition 2.8. Let $L$ be a complex finite dimensional Leibniz algebra. Then the image of a regular element of the algebra $L$ by a homomorphism $\varphi$ is a regular element of the Lie algebra $L_{\text {Lie }}$.

Proof. Let $a$ be a regular element of the algebra $L$. We shall prove that the element $\bar{a}=a+I^{\text {ann }}$ will be a regular element of the Lie algebra $L_{\text {Lie. }}$ Suppose the opposite, i.e., $\bar{a}=a+I^{\text {ann }}$ is not a regular element. Let $\bar{b}=b+I^{\text {ann }}$ be any regular element of the Lie algebra $L_{\text {Lie }}$ and $a-b \notin I^{\text {ann }}$.

Since $I^{\text {ann }}$ is an ideal, then for any $x \in L$ we have $R_{x}\left(I^{\text {ann }}\right) \subseteq I^{\text {ann }}$. It means that the matrix of the transformation $R_{x}$ has the following block form

$$
R_{x}=\left(\begin{array}{cc}
X, & 0 \\
Z_{x}, & I_{x}
\end{array}\right)
$$

in the basis $\left\{e_{1}, e_{2}, \ldots, e_{m}, i_{1}, i_{2}, \ldots, i_{n}\right\}$ of $L$, where $\left\{i_{1}, i_{2}, \ldots, i_{n}\right\}$ is the basis of $I^{\text {ann }}$. Here $X$ is the matrix of the transformation $\left.R_{x}\right|_{\left\{e_{1}, e_{2}, \ldots, e_{m}\right\}}$ and $I_{X}$ is the matrix of the transformation $\left.R_{x}\right|_{I^{\text {ann }}}$.

Let

$$
R_{a}=\left(\begin{array}{cc}
A, & 0 \\
Z_{a}, & I_{a}
\end{array}\right), \quad R_{b}=\left(\begin{array}{cc}
B, & 0 \\
Z_{b}, & I_{b}
\end{array}\right)
$$

be the matrices of the transformations $R_{a}$ and $R_{b}$ respectively.

Let $k$ (respectively $k^{\prime}$ ) be the order of the characteristic zero root of the matrix $A$ (respectively $B$ ) and $s$ and $s^{\prime}$ be the orders of the characteristic zero root of the matrices $I_{a}$ and $I_{b}$, respectively. Then we have $k^{\prime}<k, s<s^{\prime}$. 
Put

$$
\begin{gathered}
U=\left\{y \in L \backslash I^{\mathrm{ann}} \mid R_{y}=\left(\begin{array}{rr}
Y, & 0 \\
Z_{y}, I_{y}
\end{array}\right) \text { and } Y\right. \text { has the order } \\
\quad \text { of the characteristic zero root less than } k\}, \\
V=\left\{y \in L \backslash I^{\mathrm{ann}} \mid R_{y}=\left(\begin{array}{rr}
Y, & 0 \\
Z_{y}, & I_{y}
\end{array}\right) \text { and } I_{y}\right. \text { has the order } \\
\quad \text { of the characteristic zero root less than } s+1\} .
\end{gathered}
$$

Since $b \in U$ and $a \in V$ these sets are non empty.

Let us show that the set $U$ is an open subset of the set $L \backslash I^{\text {ann }}$ in the Zariski topology.

Let $Y$ have the order of the characteristic zero root less than $k$. Then $Y^{k}$ has the rank greater than $n-k$. It means that there exists a non-zero minor of the order $n-k+1$. In other words, there exists a non-zero polynomial of structural constants of the algebra $L$, hence the set $U$ is open in the Zariski topology in the subset of the set $L \backslash I^{\text {ann }}$.

One can similarly prove that the set $V$ is open in $L \backslash I^{\text {ann }}$. It is not difficult to check that the sets $U$ and $V$ are dense in $L \backslash I^{\text {ann }}$. Therefore, there exists an element $y \in U \cap V$ such that $Y$ has the order of characteristic zero root less than $k$ and $I_{y}$ has the order of the characteristic zero root less than $s+1$. Thus, for this element $y$ the order of characteristic zero root is not greater than $k+s-1$, i.e., the rank of the algebra $L$ is less than $k+s$ and we obtain a contradiction to the assumption that $\bar{a}$ is not a regular element of the Lie algebra $L_{\mathrm{Lie}}$.

Let $L$ be a Leibniz algebra with a basis $\left\{e_{1}, e_{2}, \ldots, e_{n}\right\}$ over a field $F$. Let $\xi_{1}, \xi_{2}, \ldots, \xi_{n}$ be independent variables and let $P=F\left(\xi_{1}, \xi_{2}, \ldots, \xi_{n}\right)$ be the field of rational functions of $\xi_{i}$. We construct an extension of $P$ putting $L_{P}=P e_{1}+P e_{2}+$ $\cdots+P e_{n}$

The following definition and its comments are step by step modifications of the Lie algebras case and they are included for the sake of completeness.

Definition 2.9. An element $x=\sum_{i=1}^{n} \xi_{i} e_{i}$ of the algebra $L_{P}$ is called a generic element of the algebra $L$ and the characteristic polynomial $f_{x}(\lambda)$ of the transformation $R_{x}$ in $L_{P}$ is called characteristic polynomial of the Leibniz algebra $L$.

If we take the basis $\left\{e_{1}, e_{2}, \ldots, e_{n}\right\}$ of $L_{P}$ then $\left[e_{i}, x\right]=\sum_{j=1}^{n} \rho_{i j} e_{j}$, where $i=$ $1, \ldots, n$ and $\rho_{i j}$ are homogenous functions of degree 1 with respect to $\xi_{k}$. Then

$$
f_{x}(\lambda)=\operatorname{det}\left(\lambda 1-R_{x}\right)=\lambda^{n}-\tau_{1}(\xi) \lambda^{n-1}+\tau_{2}(\xi) \lambda^{n-2}-\cdots+(-1)^{l} \tau_{n-l}(\xi) \lambda^{l},
$$

where $\tau_{i}(\xi)$ are homogenous polynomials of degree $i$ in the variables $\xi_{i}, \xi=\left\{\xi_{1}, \ldots, \xi_{n}\right\}$ and $\tau_{n-l}(\xi) \neq 0$, where $\tau_{n-l+k}(\xi)=0$ for $k>0$. Since $x \neq 0$ and $R_{x}$ is a degenerated operator, it follows that $l>0$ and $\operatorname{det} R_{x}=0$. 
The value of the characteristic polynomial on an arbitrary element $a=\sum_{i=1}^{n} \alpha_{i} e_{i}$ of the algebra $L$ is obtained by specialization of $\xi_{i}=\alpha_{i}, i=1, \ldots, n$ in equation $\left(^{*}\right)$. Therefore it is evident that the order of zero root of the characteristic transformation $R_{a}$ is not less than $l$. On the other hand, if $F$ is an infinite field then, since the polynomial $\tau_{n-l}(x)$ is different from zero in the polynomial algebra $\Phi\left[\xi_{1}, \xi_{2}, \ldots, \xi_{n}\right]$, we can choose $\xi_{i}=\alpha_{i}$ such that $\tau_{n-l}(\alpha) \neq 0$. Then the transformation $R_{a}$ for the element $a=\sum_{i=1}^{n} \alpha_{i} e_{i}$ has exactly $l$ characteristic roots which are equal to zero and therefore $a$ is regular. Thus in the case of an infinite field the element $a$ is regular if and only if $\tau_{n-l}(\alpha) \neq 0$. In this sense "almost all" elements of the algebra $L$ are regular (i.e., they form an open set in Zariski topology).

The above statement depends on the choice of the basis $(e)$. However, it is easy to observe what happens when we pass to another basis $\left(f_{1}, f_{2}, \ldots, f_{n}\right)$, where $f_{i}=\sum_{j=1}^{n} \mu_{i j} e_{j}$. If $\eta_{1}, \eta_{2}, \ldots, \eta_{n}$ are independent variables then $y=\sum_{i=1}^{n} \eta_{i} f_{i}=$ $\sum_{i=1}^{n} \eta_{i} \mu_{i j} e_{j}$. Therefore the characteristic polynomial $f_{y}(\lambda)$ is obtained from polynomial $f_{x}(\lambda)$ by substitution $\xi_{j} \rightarrow \sum_{i=1}^{n} \eta_{i} \mu_{i j}$ in its coefficients.

\section{Some properties of weight spaces of Leibniz algebras and Cartan's criterion of solvability}

In order to define a weight module over a Leibniz algebra we need the definition of the right representation of a Leibniz algebra.

Definition 3.1. A vector space $M$ is said to be a right representation of a Leibniz algebra $L$ if an action: $[\cdot, \cdot]: M \times L \rightarrow M$ is defined, which satisfies the condition

$$
[m,[x, y]]=[[m, x], y]-[[m, y], x]
$$

for any $x, y \in L, m \in M$.

Note that this definition agrees with the definition of symmetric representation in $[10]$.

Observe that $M$ has natural right $L$-module structure (in the Lie sense) and below we shall think about $M$ in that sense.

A map $a \rightarrow \alpha(a)$ from an algebra $L$ into the field $F(\alpha: L \rightarrow F)$ is called weight of the right module $M$ if there exists a non zero $x \in M$ such that $(a-\alpha(a) 1)^{k}(x)=0$, i.e., $\left[x,(a-\alpha(a) 1)^{k}\right]=0$ for some $k \in N$.

The set of vectors which satisfy this condition and the zero vector form the subspace $M_{a}$ which is called weight subspace (weight subspace with the weight $\alpha$ ) corresponding to the weight $\alpha$.

Let $L$ be a Leibniz algebra and $M$ be a weight subspace over the algebra $L$ with respect to the weight $\alpha$. Then for each element $x \in M$ one has $\left[x,(a-\alpha(a) 1)^{k}\right]=0$ if $k$ is sufficiently large. Moreover, if $\operatorname{dim} M=n$, then the polynomial $(\lambda-\alpha(a))^{n}$ is the characteristic polynomial of the endomorphism $a$. Therefore $\left[x,(a-\alpha(a) 1)^{n}\right]=0$ for any $x \in M$. 
Consider the contradredient (conjugated) right module $M^{*}$ over a Leibniz algebra satisfying the condition

$$
\left\langle[x, a], y^{*}\right\rangle+\left\langle x,\left[y^{*}, a^{*}\right]\right\rangle=0,
$$

where $x \in M, y^{*} \in M^{*}, a^{*}$ from representation which corresponds to the right module $M^{*}$ and $\left\langle x, y^{*}\right\rangle$ denote the value of linear function $y^{*}$ at the vector $x$.

It is clear that $\left\langle\alpha(a) x, y^{*}\right\rangle+\left\langle x, \alpha(a) y^{*}\right\rangle=0$.

Adding these equalities we obtain

$$
\left\langle[x,(a-\alpha(a) 1)], y^{*}\right\rangle+\left\langle x,\left[y^{*},\left(a^{*}+\alpha(a) 1\right)\right]\right\rangle=0 .
$$

By repeating this procedure, we obtain the equality

$$
\left\langle\left[x,(a-\alpha(a) 1)^{k}\right], y^{*}\right\rangle+\left\langle x,\left[y^{*},\left(a^{*}+\alpha(a) 1\right)^{k}\right]\right\rangle=0 .
$$

If $k=n$ then $\left[x,(a-\alpha(a) 1)^{n}\right]=0$ for any $x$ and thus $\left\langle x,\left[y^{*},\left(a^{*}+\alpha(a) 1\right)^{n}\right]\right\rangle=0$. Therefore $\left[y^{*},\left(a^{*}+\alpha(a) 1\right)^{n}\right]=0$ for any $y^{*} \in M^{*}$. This shows that $M^{*}$ is a weight module with the weight $-\alpha$.

Thus, we have proved the following proposition.

Proposition 3.2. If $M$ is a weight module over a Leibniz algebra with the weight $\alpha$, then the contradredient module $M^{*}$ is a weight module with weight $-\alpha$.

Proposition 3.3. If $M$ and $R$ are weight modules over a Leibniz algebra with the weight $\alpha$ and $\beta$ respectively, then $B=M \otimes R$ is a weight module with weight $\alpha+\beta$.

Proof. Proposition 3.3 is proved similarly as the corresponding proposition in the Lie algebras case (see proposition 4, page 63 in [9]).

Definition 3.4. A nilpotent Leibniz algebra $L$ of linear transformations is called $a$ split algebra if the characteristic roots of each element of $A \in L$ is contained in the basic field.

Let $L$ be a Leibniz algebra, $\mathfrak{I}$ be a nilpotent subalgebra and $M$ be a left module over $L$ (and also over $\mathfrak{I}$ ).

Suppose that $M=L$ and $R(\mathfrak{I})$ is a nilpotent split Lie algebra. From theorem 1.7 we have that $L=L_{\alpha} \oplus L_{\beta} \oplus \cdots \oplus L_{\delta}$, where $\alpha, \beta, \ldots, \delta$ are maps from the subalgebra $R(\mathfrak{I})$ into $F$ such that if $x_{\nu} \in L_{\nu}$, then $\left(R_{h}-\nu\left(R_{h}\right) 1\right)^{m}\left(x_{\nu}\right)=0$ for some $m=m(\nu)$, where $\nu \in\{\alpha, \beta, \ldots, \delta\}$. The weights $\alpha, \beta, \ldots, \delta$ are called roots of the algebra $L$ with respect to the subalgebra $\mathfrak{I}$.

Proposition 3.5. $\left[L_{\alpha}, L_{\beta}\right] \subseteq L_{\alpha+\beta}$ if $\alpha+\beta$ is a root of the Leibniz algebra $L$ with respect to $R(\mathfrak{I})$; otherwise $\left[L_{\alpha}, L_{\beta}\right]=0$. 
Proof. The elements of $\left[L_{\alpha}, L_{\beta}\right]$ have the form $\sum_{i}\left[x_{\alpha}^{(i)}, y_{\beta}^{(i)}\right]$ where $x_{\alpha}^{(i)} \in L_{\alpha}, y_{\beta}^{(i)} \in L_{\beta}$. From the characteristic property of the tensor product of two spaces it follows that there exists a linear map $\pi: L_{\alpha} \otimes L_{\beta} \rightarrow\left[L_{\alpha}, L_{\beta}\right]$ such that $\pi\left(\sum_{i} x_{\alpha}^{(i)} \otimes y_{\beta}^{(i)}\right)=$ $\sum_{i}\left[x_{\alpha}^{(i)}, y_{\beta}^{(i)}\right]$. We show that $\pi$ is actually a homomorphism of $R(\mathfrak{I})$-modules.

Let $R_{h} \in R(\mathfrak{I})$ then using the Leibniz identity we obtain the following chain of equalities:

$$
\begin{aligned}
R_{h}\left(x_{\alpha} \otimes y_{\beta}\right) & =R_{h}\left(x_{\alpha}\right) \otimes y_{\beta}+x_{\alpha} \otimes R_{h}\left(y_{\beta}\right) \\
& =\left[x_{\alpha}, h\right] \otimes y_{\beta}+x_{\alpha} \otimes\left[y_{\beta}, h\right] \rightarrow\left[\left[x_{\alpha}, h\right], y_{\beta}\right]+\left[x_{\alpha},\left[y_{\beta}, h\right]\right] \\
& =R_{h}\left(\left[x_{\alpha}, y_{\beta}\right]\right) .
\end{aligned}
$$

On the other hand, the image of the element $x_{\alpha} \otimes y_{\beta}$ under the homomorphism $\pi$ is $\left[x_{\alpha}, y_{\beta}\right]$. So, we prove that $\left[L_{\alpha}, L_{\beta}\right]$ is a homomorphic image of the module $L_{\alpha} \otimes L_{\beta}$. Moreover, $L_{\alpha} \otimes L_{\beta}$ is a weight module with the weight $\alpha+\beta$. But from the definition it is clear that the homomorphic image of the weight module with the weight $\beta$ is either 0 or a weight module with the weight $\beta$.

Let $L$ be a finite dimensional Leibniz algebra over an algebraically closed field $F$ and $\mathfrak{I}$ be a nilpotent subalgebra of $L$. Let $L=L_{\alpha} \oplus L_{\beta} \oplus \cdots \oplus L_{\delta}$ be a decomposition of module $L$ into the direct sum of weight submodules with respect to $\mathfrak{I}$.

Suppose that $\mathfrak{I}$ is a Cartan subalgebra. Then it is not difficult to see that $\mathfrak{I}=L_{0}$, where $L_{0}$ is the root module corresponding to the root 0 .

We have also the equality $[L, L]=\sum\left[L_{\alpha}, L_{\beta}\right]$, where the sum is taken over all roots $\alpha, \beta$. From this we obtain $L_{0} \cap L^{2}=\mathfrak{I} \cap L^{2}=\sum\left[L_{\alpha}, L_{-\alpha}\right]$, where summation is made over all $\alpha$, such that $-\alpha$ is also a root (in particular $\alpha=0$ ).

Definition 3.6. The form $f(a, b)=\operatorname{tr}\left(R_{a} R_{b}\right)$ for $a, b \in L$ is called the Killing form of the Leibniz algebra $L$.

A bilinear form $f(a, b)$ on $L$ satisfying the condition

$$
f([a, c], b)+f(a,[b, c])=0
$$

is called an invariant form on $L$.

The following equalities show that the Killing form is an invariant form on Leibniz algebra:

$$
\begin{aligned}
f([a, c], b)+f(a,[b, c]) & =\operatorname{tr}\left(R_{[a, c]} R_{b}\right)+\operatorname{tr}\left(R_{a} R_{[b, c]}\right) \\
& =\operatorname{tr}\left(\left[R_{c}, R_{a}\right] R_{b}+R_{a}\left[R_{c}, R_{b}\right]\right) \\
& =\operatorname{tr}\left(\left(R_{c} R_{a}-R_{a} R_{c}\right) R_{b}+R_{a}\left(R_{c} R_{b}-R_{b} R_{c}\right)\right) \\
& =\operatorname{tr}\left(R_{c} R_{a} R_{b}-R_{a} R_{b} R_{c}\right)=\operatorname{tr}\left[R_{c}, R_{a} R_{b}\right]=0 .
\end{aligned}
$$

Note that if $f(a, b)$ is the Killing form then the set

$$
L^{\perp}=\{z \in L \mid f(a, z)=0 \text { for any } a \in L\}
$$


is an ideal of the algebra $L$.

Theorem 3.7. Let $L$ be a Leibniz algebra over an algebraically closed field of zero characteristic. Then $L$ is solvable if and only if $\operatorname{tr}\left(R_{a} R_{a}\right)=0$ for any $a \in L^{2}$.

Proof. The necessity. In proposition 1.8 put $\aleph:=R(L)$, then it is clear that the Lie algebra $R(L)$ is also solvable and therefore $[R(L), R(L)] \subseteq \Re$. And since $[R(L), R(L)]=$ $R\left(L^{2}\right)$ we have that for any $a \in L^{2}$ the operator $R_{a}$ is nilpotent, therefore $\operatorname{tr}\left(R_{a} R_{a}\right)=0$ for any $a \in L$.

The sufficiency. If we apply Cartan's criterion for Lie algebras [9] for algebra $R(L)$ and consider $L$ as $R(L)$-module, we obtain the solvability of the Lie algebra $R(L)$, but as we noted in section 1 it is equivalent to the solvability of Leibniz algebra $L$.

Acknowledgements. The second and third named authors would like to acknowledge the hospitality to the "Institut für Angewandte Mathematik", Universität Bonn (Germany).

\section{References}

[1] S. Albeverio, Sh. A. Ayupov, and B. A. Omirov, On nilpotent and simple Leibniz algebras, Comm. Algebra 33 (2005), no. 1, 159-172.

[2] Sh. A. Ayupov and B. A. Omirov, On some classes of nilpotent Leibniz algebras, Sibirsk. Mat. Zh. 42 (2001), no. 1, 18-29, i (Russian).

[3] Nilpotent properties of the Leibniz algebra $M_{n}(\mathbb{C})_{D}$, Sibirsk. Mat. Zh. 45 (2004), no. 3, 483-496 (Russian).

[4] - On Leibniz algebras, Algebra and operator theory (Tashkent, 1997), 1998, pp. 1-12.

[5] J. M. Casas and T. Pirashvili, Ten-term exact sequence of Leibniz homology, J. Algebra 231 (2000), no. 1, 258-264.

[6] A. Frabetti, Leibniz homology of dialgebras of matrices, J. Pure Appl. Algebra 129 (1998), no. 2, 123-141.

[7] Y. Gao, The second Leibniz homology group for Kac-Moody Lie algebras, Bull. London Math. Soc. 32 (2000), no. 1, 25-33.

[8] A. V. Gnedbaye, Leibniz homology of extended Lie algebras, K-Theory 13 (1998), no. 2, $169-178$.

[9] N. Jacobson, Lie algebras, Izdat. "Mir", Moscow, 1964.

[10] J.-L. Loday, Une version non commutative des algèbres de Lie: les algèbres de Leibniz, Enseign. Math. (2) 39 (1993), no. 3-4, 269-293.

[11] J.-L. Loday and T. Pirashvili, Universal enveloping algebras of Leibniz algebras and (co)homology, Math. Ann. 296 (1993), no. 1, 139-158.

[12] A. Malcev, On solvable Lie algebras, Izvestia Akad. Nauk SSSR 9 (1945), 329-356 (Russian). 